Article

\title{
Evaluation of the Toxicity and Toxicokinetics of Cereulide from an Emetic Bacillus cereus Strain of Milk Origin
}

\author{
Yifang Cui ${ }^{1}$, Yuan Liu ${ }^{1}$, Xiaoye Liu ${ }^{1}$, Xi Xia ${ }^{1}$, Shuangyang Ding ${ }^{1, *}$ and Kui Zhu ${ }^{1,2, *}$ \\ 1 Beijing Advanced Innovation Center for Food Nutrition and Human Health, College of Veterinary Medicine, \\ China Agricultural University, Beijing 100193, China; yayapi19@163.com (Y.C.); liu_yuan@cau.edu.cn (Y.L.); \\ leaf19880705@126.com (X.L.); xxia@cau.edu.cn (X.X.) \\ 2 Department of Biomedical Engineering, Duke University, Durham, NC 27708, USA \\ * Correspondence: dingsy@cau.edu.cn (S.D.), zhukcau@gmail.com (K.Z.); Tel.: +86-106-273-3857 (S.D.); \\ +86-106-273-4255 (K.Z.)
}

Academic Editor: Adam J. Ratner

Received: 13 March 2016; Accepted: 11 May 2016; Published: 6 June 2016

\begin{abstract}
Bacillus cereus is an opportunistic foodborne agent causing food poisoning and many infectious diseases. The heat-stable emetic toxin cereulide is one of the most prevalent toxins produced by pathogenic $B$. cereus, resulting in symptoms such as emesis and liver failure. In the present work, the toxicity and toxicokinetics of cereulide from an emetic B. cereus isolate (CAU45) of raw milk were evaluated. The production of cereulide was tested by a cytotoxicity test and enzyme immunoassay, and confirmed by the presence of the ces (cereulide synthetase) gene and the ultra-performance liquid chromatography tandem mass spectrometry (UPLC-MS/MS) method. All results showed that the amount and toxicity of cereulide produced by CAU45 was 7 to 15.3 folds higher than the reference emetic B. cereus DSMZ 4312. Cereulide in plasma was collected at different time points after a single intravenous injection to evaluate its toxicokinetics in rabbits. The maximum concentration of cereulide was achieved in $2.6 \pm 3.4 \mathrm{~h}$ after administration, with the elimination half-life of $10.8 \pm 9.1 \mathrm{~h}$, which expands our understanding of the toxic effects of cereulide. Together, it suggests that urgent sanitary practices are needed to eliminate emetic toxins and emetic B. cereus in raw milk.
\end{abstract}

Keywords: Bacillus cereus; cereulide; toxicity; toxicokinetics; UPLC-MS/MS

\section{Introduction}

B. cereus is a spore-forming, ubiquitously opportunistic pathogen, causing various diseases including food poisoning, fulminant bacteremia, pneumonia and endophthalmitis [1]. Foodborne B. cereus causes two types of symptoms: diarrhea and emesis [2]. Two tripartite enterotoxin complexes, non-hemolytic enterotoxin (Nhe) and hemolysin BL ( $\mathrm{Hbl})$, and a single component of cytotoxin K1 (CytK1) are potentially responsible for diarrhea [3,4], whereas emetic syndrome is induced by a lipophilic cyclic dodecadepsipeptide cereulide, which previously formed in the contaminated foods. Additionally, cereulide has been reported to result in fulminate liver failure in children after ingestion of B. cereus-contaminated spaghetti and pesto $[5,6]$.

B. cereus can be found in most common food matrices such as rice, pasta and dairy products, and it represents more than $68.0 \%$ of foodborne outbreaks in all food types [7]. There is an increasing occurrence of $B$. cereus foodborne outbreaks. For example, B. cereus was responsible for $13.4 \%$ of bacterial foodborne outbreaks, ranking as the second among the most frequent bacterial causes of foodborne disease in Chinese inland provinces [8]. In fact, the number of B. cereus contaminations 
is highly underestimated, because foodborne B. cereus disease is mild and self-limiting and is consequently not reported by patients. The presence of cereulide or emetic B. cereus in foods is a severe hazard for consumer safety. The heat-stable, proteolytically resistant and preformed cereulide is still active and can cause intoxication after the heat treatment in food processing and passing through the intestinal tract [9]. For instance, emetic B. cereus strains have been detected not only in farinaceous foods commonly correlated with cereulide intoxication but also in cheese, salads and meat products [10]. B. cereus sensu stricto isolates have also been shown to produce Nhe and $\mathrm{Hbl}$ enterotoxins in milk and dairy products in China [11,12]; however, the presence of cereulide in milk or related products has not been reported yet.

Cereulide is synthesized by non-ribosomal peptide synthetases (NRPS) encoded by the ces gene cluster [13]. As an ionophore with high affinity to $\mathrm{K}^{+}$, cereulide can interfere the electrochemical potential gradient on lipid membranes and inhibit mitochondrial activity $[14,15]$. Although cereulide has been shown to induce emesis in animal models [16,17], the toxicokinetics of cereulide remain unclear. In this work, we firstly characterized an emetic B. cereus isolate from raw milk in a local dairy farm of Beijing, China, based on the presence of the ces gene and a marker protein of cereulide (CBP) by immunoassay. Secondly, we quantified the production of cereulide by UPLC-MS/MS, and evaluated its virulence with a cytotoxicity test. Lastly, the toxicokinetics of cereulide were evaluated in rabbits.

\section{Results}

\subsection{Characterization of B. cereus CAU45}

The B. cereus CAU45 strain was tested for the presence of four main toxin genes, $n h e, h b l, c y t K 1$ and ces. The presence of $n h e A, n h e B$ and $n h e C$, and ces genes was found in B. cereus CAU45 and confirmed by sequence analysis, as shown in Figure 1a, and the ces gene cluster was located on a plasmid of $B$. cereus CAU45. Subsequently, the ability of Nhe and cereulide production was tested by immunoassays and cytotoxicity tests. A low level of NheA and no NheB was detected by monoclonal antibodies (mAbs) $1 \mathrm{~A} 8$ and $1 \mathrm{E} 11$ in B. cereus CAU45, respectively (Figure $1 \mathrm{~b}$ ). However, the titer of CBP in B. cereus CAU45 $(270 \pm 15)$ was 8.7 folds higher than that in $B$. cereus DSMZ $4312(31 \pm 4)$ (Figure $1 b, P=0.0097)$. This was consistent with the cytotoxicity result that the cytotoxic titer of cereulide from B. cereus CAU45 (2384 \pm 57 ) was 15.3 times higher than m DSMZ $4312(156 \pm 15)$ in HEp-2 cells (Figure 1c, $P=0.0003$ ). Lastly, the production of cereulide in B. cereus CAU45 was quantified by UPLC-MS/MS assay. The amount of cereulide in B. cereus CAU45 (Figure 1e) was about $13.3 \mu \mathrm{g} / \mathrm{mg}$ biomass (wet weight) with the proportion of $93.8 \%$ in the crude extracts and it was seven times higher than in the reference strain B. cereus DSMZ 4312, which was about $1.9 \mu \mathrm{g} / \mathrm{mg}$ biomass (wet weight) (Figure 1d). 

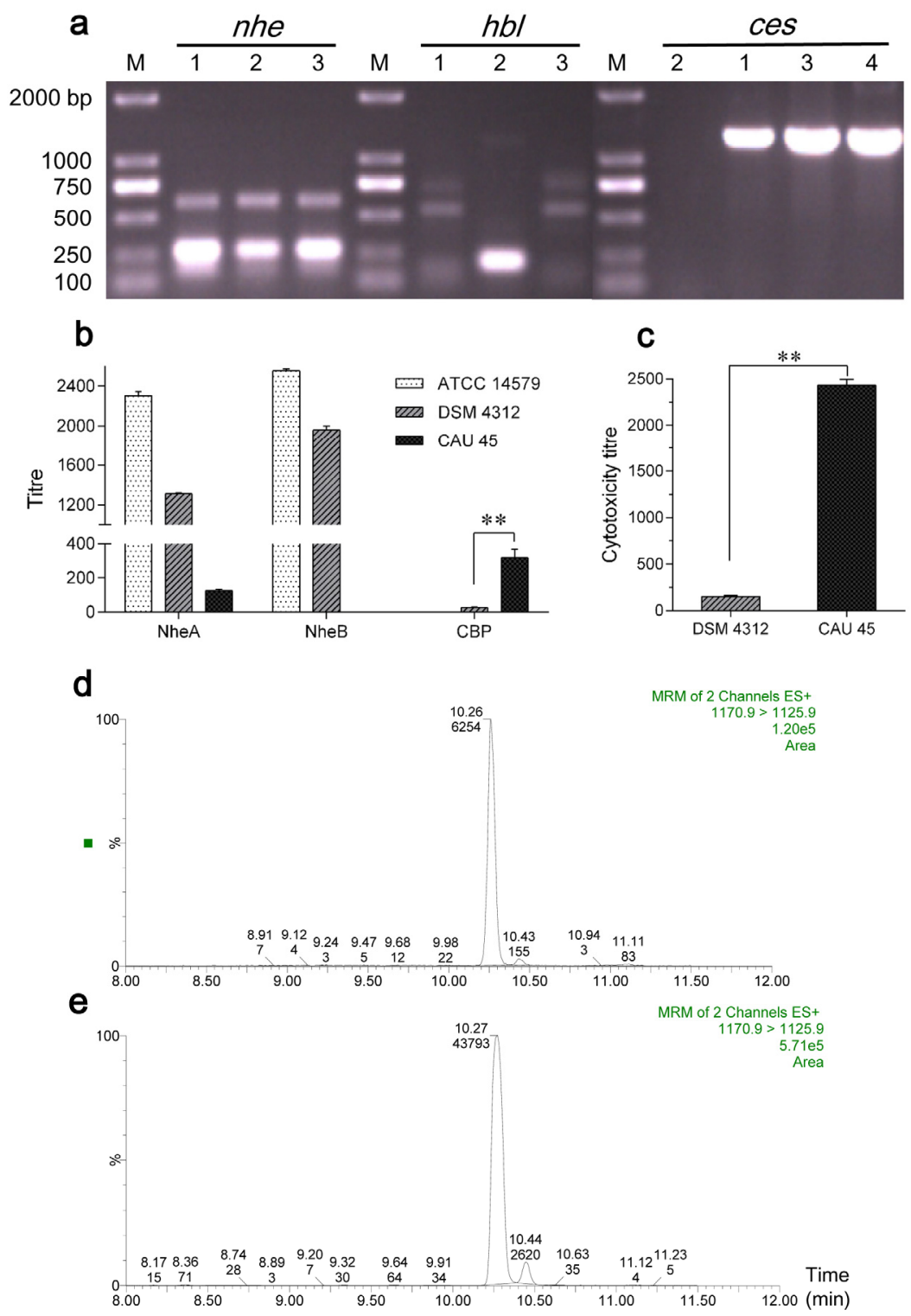

Figure 1. Characterization of the toxin profiles of raw milk isolated B. cereus CAU45 strain. (a) Identification of the toxin genes $n h e, h b l$ and ces by PCR assays. The presence of $n h e A, n h e B$ and $n h e C, h b l A$ and $h b l D$, and ces was tested. The amplicons of targets were sequenced and submitted for sequence analysis. Lanes 1-4 were the reference strains of B. cereus DSMZ 4312 containing nhe and ces genes (1), B. cereus ATCC 14579 containing nhe and $h b l$ genes (2), and raw milk isolated B. cereus CAU45 (3) and its plasmid (4). M, size marker (TransGen Biotech, Beijing, China); (b) The presence of NheA, NheB, and maker protein of cereulide (CBP) was detected based on specific monoclonal antibodies. For B. cereus CAU45, low titer of NheA was detected, and no NheB was found. The presence of CBP was found in B. cereus DSMZ 4312 and CAU45 strains, and showed significant difference $(n=3$, ** $P=0.0097)$; (c) Cytotoxicity of cereulide was tested in HEp-2 cells. Means \pm SD were presented throughout $\left(n=3,{ }^{* *} P=0.0003\right) ;(\mathbf{d}, \mathbf{e})$ UPLC chromatograms of cereulide in B. cereus DSMZ 4312 (d) and CAU45 strains (e).

\subsection{Quantification of Cereulide in Plasma by UPLC-MS/MS}

Representative chromatograms of blank plasma and plasma spiked with cereulide (50 ng/mL) and valinomycin $(10 \mathrm{ng} / \mathrm{mL})$ are shown in Figure 2. The retention times of cereulide and valinomycin were $2.14 \mathrm{~min}$ and $4.00 \mathrm{~min}$. There were no interfering peaks at the retention times of targets of interest 
in the blank plasma (Figure 2a). The calibration curve of cereulide showed good linearity in the range from $1.5 \mathrm{ng} / \mathrm{mL}$ to $1000 \mathrm{ng} / \mathrm{mL}$, with $r=0.9992$. The limit of quantitation (LOQ, $/ \mathrm{N}=10$ ) was $1.5 \mathrm{ng} / \mathrm{mL}$ for cereulide in plasma. Additionally, the recoveries of cereulide spiked at three different concentrations (10, 50 and $100 \mathrm{ng} / \mathrm{mL}$ ) were $98.7 \%, 87.8 \%$ and $104.4 \%$, respectively. Together it indicated that the developed UPLC-MS/MS method in the present work was suitable for analyzing cereulide in plasma samples.
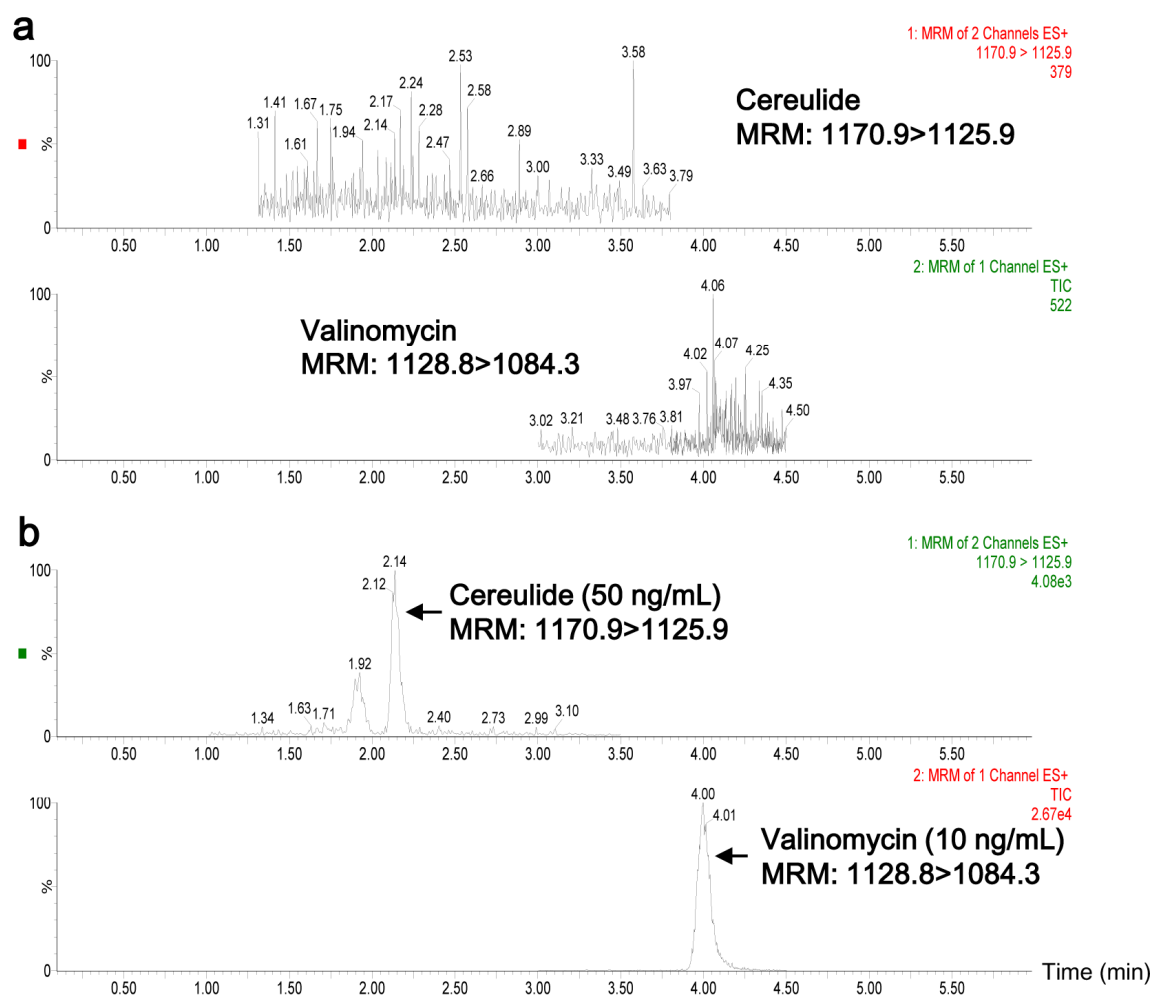

Figure 2. UPLC-MS/MS chromatograms of cereulide and valinomycin in rabbit plasma. (a) Blank plasma samples and (b) plasma sample spiked with extracted cereulide $(50 \mathrm{ng} / \mathrm{mL})$ from B. cereus CAU45 strain and valinomycin $(10 \mathrm{ng} / \mathrm{mL})$.

\subsection{Toxicokinetics of Cereulide in Rabbits}

A total number of eight rabbits (half female and half male) were used to evaluate the toxicokinetics of cereulide after a single administration of $5 \mu \mathrm{g}$ cereulide. All plasma samples at different time points were collected, and the concentrations of cereulide were quantified by UPLC-MS/MS assay. The dynamic curve of cereulide in the plasma of rabbits is shown in Figure 3a, and toxicokinetic parameters were calculated using a non-compartmental model as shown in Table 1. Cereulide was detected in plasma within $0.2 \mathrm{~h}$ after a single intravenous injection and reached its maximum concentration of $40.8 \pm 21.6 \mathrm{ng} / \mathrm{mL}$. The concentration of cereulide dropped down until $1 \mathrm{~h}$ and rose thereafter. Following a short stationary phase from $4 \mathrm{~h}$ to $8 \mathrm{~h}$, the concentration of cereulide decreased gradually. No cereulide could be detected $32 \mathrm{~h}$ later. Additionally, no cereulide was detected in the rabbits treated by $0.9 \%$ saline-methanol $(10: 90, v / v)$ based on our established UPLC-MS/MS method. 

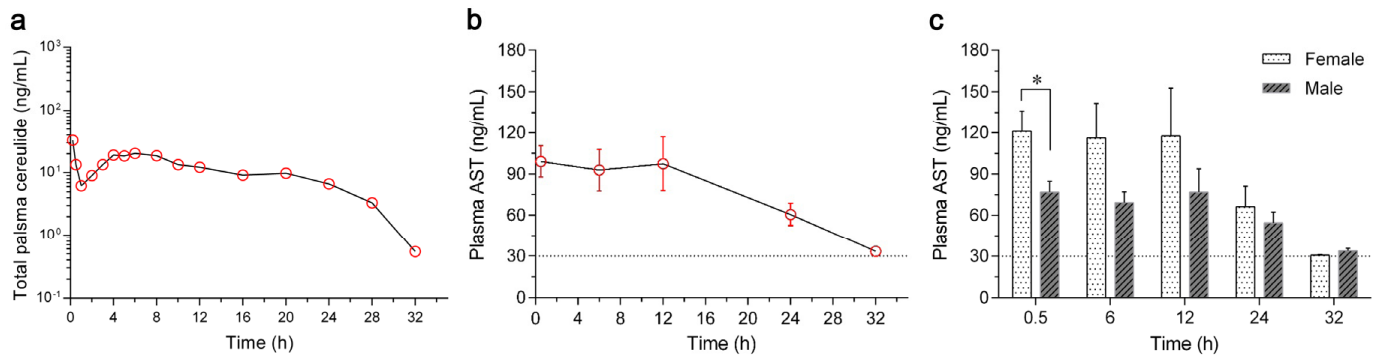

Figure 3. Concentrations of cereulide and aspartate aminotransferase (AST) in the plasma of rabbits. (a) A total number of eight rabbits (four female and four male) were used, with the weights of $2.0 \pm 0.3 \mathrm{~kg} /$ rabbit. Cereulide extracted from the emetic B. cereus CAU45 strain was administrated through ear vein with the dose of $5 \mu \mathrm{g} / \mathrm{rabbit}$, and plasma samples were collected at time points of $0,0.2,0.5,1,2,3,4,5,6,8,10,12,16,20,24,28$ and $32 \mathrm{~h}$. Each plasma sample was spiked with valinomycin $(10 \mathrm{ng} / \mathrm{mL})$ as an internal standard to quantify cereulide by UPLC-MS/MS; (b) Concentrations of AST in the plasma were detected by EIA (Beyotime Biotechnology, Beijing, China), at the time points of $0.5,6,12,24$ and $32 \mathrm{~h}$ after a single intravenous injection of cereulide in rabbits. The concentrations of AST in plasma were below $30 \mu \mathrm{g} / \mathrm{mL}$ in negative controls, as indicated by the dash line. Means \pm SD were presented throughout $(n=8)$; (c) Concentration dynamics of plasma AST in female and male rabbits. The AST concentrations of female rabbits were higher $\left(n=4,{ }^{*} P=0.0345\right)$ than the males at the time point of $0.5 \mathrm{~h}$, and other time points showed no significant difference.

Table 1. Toxicokinetic parameters of a single intravenous injection of cereulide in rabbits.

\begin{tabular}{cccc}
\hline Toxicokinetic Parameters * & Median & Range & Mean \pm SD \\
\hline AUC $(\mathrm{h} \times \mathrm{ng} / \mathrm{mL})$ & 309.3 & $81.2-851.1$ & $320.9 \pm 240.1$ \\
$\mathrm{AUC} \infty(\mathrm{h} \times \mathrm{ng} / \mathrm{mL})$ & 330.5 & $126.0-853.9$ & $390.3 \pm 257.9$ \\
Cl $(\mathrm{mL} / \mathrm{h})$ & 15.5 & $5.9-39.7$ & $19.1 \pm 12.8$ \\
C $_{\max }(\mathrm{ng} / \mathrm{mL})$ & 33.8 & $13.3-78.1$ & $40.8 \pm 21.6$ \\
HL_Lambda_z $(\mathrm{h})$ & 6.9 & $1.9-24.5$ & $10.8 \pm 9.1$ \\
Lambda_z $(1 / \mathrm{h})$ & 0.1 & $0.03-0.4$ & $0.1 \pm 0.1$ \\
MRTlast $(\mathrm{h})$ & 10.6 & $4.5-13.4$ & $9.6 \pm 2.9$ \\
$\mathrm{~T}_{\max }(\mathrm{h})$ & 0.2 & $0.2-8.0$ & $2.6 \pm 3.4$ \\
Vd $(\mathrm{mL})$ & 208.1 & $16.3-1254.5$ & $320.1 \pm 399.6$ \\
\hline
\end{tabular}

* The toxicokinetic parameters were analyzed by the commercial pharmacokinetic program Phoenix WinNonlin 5.2.1 (Certara). AUC, area under the curve for the plasma concentration until the last time point; $\mathrm{AUC}_{\infty}$, area under the curve until infinity; $\mathrm{Cl}$, clearance; $\mathrm{C}_{\max }$, maximum concentration after intravenous administration; HL_Lambda_z, elimination half-life; Lambda_z, elimination rate constant; MRTlast, mean retention time until the last time point; $\mathrm{T}_{\max }$, time to peak concentration; $\mathrm{Vd}$, volume of distribution.

Liver damage in rabbits triggered by cereulide was evaluated by the concentration of aspartate aminotransferase (AST) in plasma. Comparing with the range of AST in the control (from $15.0 \mathrm{ng} / \mathrm{mL}$ to $30.0 \mathrm{ng} / \mathrm{mL})$, a sharp increase in the AST concentration $(99.1 \pm 32.2 \mathrm{ng} / \mathrm{mL})$ was found $0.5 \mathrm{~h}$ after the injection of cereulide and it lasted for $12 \mathrm{~h}(97.3 \pm 55.3 \mathrm{ng} / \mathrm{mL})$, as shown in Figure 3b. Liver function recovered, with the concentration of AST returning to the normal level $32 \mathrm{~h}$ after administration. In addition, the concentrations of AST in female rabbits were higher than those in males (Figure $3 c$ ), especially at the time point of $0.5 \mathrm{~h}(P=0.0345)$.

\section{Discussion}

The increased incidence of $B$. cereus foodborne outbreaks is greatly associated with different types of foods [18-22]. Due to the formation of notorious endospores and the previously formed and heat-stable cereulide, emetic $B$. cereus can enter and survive during food production, processing and storage processes at various points, evoking vomiting in a few hours after ingestion [23]. Much more attention should be paid to the food matrices, especially the ones supporting the production of cereulide. 
Until now, emetic B. cereus group isolates have been widely reported in many countries $[10,24,25]$, but only a few emetic $B$. cereus group strains were reported in China. For example, two emetic B. cereus strains and two emetic $B$. weihenstephanensis strains have been reported from fermented black beans and ice creams, respectively [12,19], and the prevalence of emetic B. cereus strains in these products remains unclear. In the present work, we firstly characterized an emetic B. cereus sensu stricto isolate from a local dairy farm of Beijing, China, with a high ability of cereulide production (Figure 1). Compared with the ces gene cluster of B. cereus plasmid pBCE4810 (GenBank accession No. NG_036207.1) [26], the ces sequence of $B$. cereus CAU45 showed a $99.9 \%$ identity. The production of cereulide can be also influenced by various factors [23], and further research is still needed to elucidate the enhanced production of cereulide. Additionally, the disability to express intact Nhe complex in B. cereus CAU45 makes it a great candidate to study the dissemination of emetic $B$. cereus and the function of cereulide in contaminated foods using animal models, without the influence of expressed enterotoxin Nhe as in most pathogenic B. cereus strains [27].

The toxicokinetics of extracted cereulide were evaluated in rabbits by the UPLC-MS/MS method. The concentration of cereulide in plasma rapidly decreased in the first hour after a single dose of intravenous injection (Figure 3a). Based on toxicokinetic analysis, we found that cereulide had a short elimination half-life (HL_Lambda_z) of $10.8 \pm 9.1 \mathrm{~h}$, with the mean retention time (MRTlast) of $9.6 \pm 2.9 \mathrm{~h}$ and small volume of distribution $(\mathrm{Vd})$ of $320.1 \pm 399.6 \mathrm{~mL}$, shown in Table 1 . All data indicated that cereulide was eliminated or metabolized very fast in rabbits, which is consistent with the observation that the symptoms of the cereulide intoxication disappear after $6 \mathrm{~h}$ of food digestion [28].

The rapid increase of the AST concentration in plasma (Figure 3b) indicated the fast transfer of cereulide from blood to liver, resulting in liver injury with the liver losing its function. The time to recovery of the concentration of AST to normal range in rabbits was $32 \mathrm{~h}$. AST reached its maximum concentration after $12 \mathrm{~h}$ of cereulide treatment in rabbits; however, it took $48 \mathrm{~h}$ to achieve the maximum concentration in mice [29]. Altogether, the different symptoms in various animal models might be ascribed to their physiological characters, or different doses of cereulide, or alternate routes of administration used in each animal model. In addition, higher levels of AST were also observed in female rabbits than male, indicating a gender difference may affect the metabolism of cereulide in rabbits as well.

In summary, we isolated a high virulent emetic B. cereus sensu stricto mutant strain, CAU45. This strain carried nhe and ces genes without the expression of intact Nhe complex. High production of cereulide in B. cereus CAU45 was correlated with its high cytotoxicity. Moreover, we studied the toxicokinetics of cereulide in rabbits after a single intravenous injection and monitored the damage in their livers. The toxicokinetic parameters might imply the rapid appearance of clinical symptoms. Interestingly, the toxic effect caused by cereulide showed a potential gender difference in rabbits. Altogether, the presence of emetic $B$. cereus in raw milk suggests that urgent sanitary practices are needed to eliminate emetic toxin and emetic $B$. cereus, both for improving the quality of milk and dairy products and reducing the incidence of $B$. cereus-associated diseases.

\section{Materials and Methods}

\subsection{Bacterial Strains and Materials}

B. cereus CAU45 was isolated from a raw milk sample in one local dairy farm of Beijing, China. B. cereus DSMZ 4312 was used as a reference strain for cereulide and Nhe, while B. cereus ATCC 14579 was chosen as a reference strain for Nhe and $\mathrm{Hbl}$. All the monoclonal antibodies used for the detection of NheA and NheB, and CBP were as previously described [27]. HEp-2 cells used to evaluate the cytotoxicity of cereulide were obtained from Prof. Xiaojia Wang at China Agricultural University. Valinomycin purchased from Sigma-Aldrich (St. Louis, MO, USA) was used as an internal standard by UPLC-MS/MS test. 


\subsection{Isolation and Identification the Emetic B. cereus Strain}

The isolation of B. cereus was performed according to the previous protocol [30]. Briefly, the blue/green colonies on Brilliance ${ }^{\mathrm{TM}}$ Bacillus cereus Agar (Oxoid, Basingstoke, UK) plates, after incubating at $32{ }^{\circ} \mathrm{C}$ for $24 \mathrm{~h}$, were selected as presumptive $B$. cereus strains. The positive colonies were subsequently passaged on brain heart infusion (BHI, Land Bridge Technology, Beijing, China) agar plates, and single colonies were chosen for the further study.

The genomic DNA was extracted by boiled water method and the plasmid was extracted by QIAfilter Plasmid Midi Kits (QIAGEN, Duesseldorf, Germany) as described before [31]. Four main toxin genes, nhe, $h b l, c y t K 1$ and ces, were tested by PCR assays [32]. The amplicons of targets were sequenced and blasted with reference sequences by DNAMAN 8.0.5.789 software (Lynnon Biosoft, San Ramon, CA, USA). Caseinhydrolysat-glucose-yeast (CGY) medium plus 1\% glucose (Sigma-Aldrich, St. Louis, MO, USA) was used to produce Nhe [27]. The presence of Nhe and CBP was detected by enzyme immunoassays (EIAs) as previously described [27,33]. HEp-2 cells were used to evaluate the cytotoxicity of cereulide based on water-soluble tetrazolium salt-1 (WST-1, Roche, Basel, Switzerland) method [34].

\subsection{Extraction of Cereulide}

Cereulide was extracted from the cultures of B. cereus CAU45 and DSMZ 4312 on trypticase soy agar (TSA, Land Bridge Technology, Beijing, China) plates at $32{ }^{\circ} \mathrm{C}$ for 10 days. Briefly, bacterial cultures were collected and extracted with methanol (approximate $100 \mathrm{mg}$ bacterial biomass, wet weight dissolved in $1 \mathrm{~mL}$ methanol, Fisher Scientific, Waltham, PA, USA), heated at $80{ }^{\circ} \mathrm{C}$ for $30 \mathrm{~min}$. The residues were resuspended in $0.5 \mathrm{~mL}$ methanol, vortexed for $2 \mathrm{~min}$ and centrifuged at $13,000 \mathrm{~g}$ for $5 \mathrm{~min}$. The supernatants were evaporated to dryness with a flow of nitrogen gas, and dissolved in methanol. All the extracts were stored in dark glass vials at $-20^{\circ} \mathrm{C}$.

\subsection{Cereulide Treated Rabbits}

This animal study was approved by the Institutional Animal Care and Use Committee at the China Agricultural University (SYXK (2012-0037), 28 December 2012), and all the operations were conducted according to the biosafety procedures. Eight adult New Zealand White rabbits (four female and four male) with weights of $2.0 \pm 0.3 \mathrm{~kg}$ were used in this study. A single intravenous dose of $5 \mu \mathrm{g}$ cereulide dissolved in $0.9 \%$ saline-methanol $(10: 90, v / v$; the amount of methanol injected to rabbit was about $1.8 \mathrm{~g} / \mathrm{kg}$ body weight) was administrated. Two other rabbits (one female and one male) were treated by $0.9 \%$ saline-methanol $(10: 90, v / v)$ as control. Blood samples were collected after injection at different time points $0,0.2,0.5,1,2,3,4,5,6,8,10,12,16,20,24,28,32 \mathrm{~h}$. All samples were centrifuged at $4{ }^{\circ} \mathrm{C}, 2000 \mathrm{~g}$ for $10 \mathrm{~min}$. Plasma from each sample was collected and stored at $-80{ }^{\circ} \mathrm{C}$.

\subsection{Quantification of Cereulide in Plasma by UPLC-MS/MS}

The plasma samples were thawed to room temperature before analysis. Then $200 \mu \mathrm{L}$ of each sample was spiked with $10 \mathrm{ng} / \mathrm{mL}$ valinomycin (Sigma-Aldrich, St. Louis, MO, USA) as internal standards in a $1.5 \mathrm{~mL}$ tube (Axygen, Union City, CA, USA). The extraction steps were as below. First $1 \mathrm{~mL}$ acetonitrile (Fisher Scientific, Waltham, PA, USA) was added to each sample and vortexed for $3 \mathrm{~min}$. After centrifugation at 13,000 $\mathrm{g}$ for $10 \mathrm{~min}$, the supernatants were transferred into new tubes. The extraction steps were repeated once and the supernatants were combined. The supernatants were evaporated under a flow of nitrogen gas at $50{ }^{\circ} \mathrm{C}$. The dry samples were reconstituted with $500 \mu \mathrm{L}$ methanol and water with the ratio of 9:1 $(v / v)$, vortexed for $3 \mathrm{~min}$ and filtered through $0.22 \mu \mathrm{m}$ filter membrane (PALL, Port Washington, NY, USA) before UPLC-MS/MS analysis.

The presence of cereulide was confirmed in all extracts and quantified by UPLC-MS/MS as described before [35]. UPLC-MS/MS (Waters, Milford, MA, USA) equipped with a RP C18 column $(50 \mathrm{~mm} \times 2.1 \mathrm{~mm}, 1.7 \mu \mathrm{m}$, Acquity UPLC, Milford, MA, USA) was used. A gradient program was 
applied with the mobile phase consisting of solvent A ( $0.1 \%$ formic acid (Dikma, Lake Forest, CA, USA) in water) and solvent B (0.1\% formic acid in acetonitrile) as follows: $98 \%-10 \% \mathrm{~A}(0-1 \mathrm{~min})$, $10 \%-0 \%$ A (1-3 min), 0\%-98\% A (3-5 min). The flow rate was set at $0.3 \mathrm{~mL} / \mathrm{min}$ with the injection volume of $10 \mu \mathrm{L}$. A positive electrospray ionization $\left(\mathrm{ESI}^{+}\right)$mode was chosen to analyze the transitions of $m / z 1170.9 \rightarrow 1125.9$ for cereulide and $m / z 1128.8 \rightarrow 1084.3$ for valinomycin, by multiple reaction monitoring (MRM) method. The cone voltages for cereulide and valinomycin were $10 \mathrm{~V}$ and $60 \mathrm{~V}$, and collision energy were $20 \mathrm{eV}$ and $37 \mathrm{eV}$, respectively. All data were acquired and processed by software Masslynx 4.1 (Waters, Milford, MA, USA, 2005). The toxicokinetic parameters of cereulide were analyzed by the commercial pharmacokinetic program Phoenix WinNonlin 5.2.1 (Certara, Princeton, NJ, USA).

\subsection{AST Measurement}

Plasma samples at time points of $0.5,6,12,24$ and $32 \mathrm{~h}$ were evaluated concentrations of AST by indirect EIA according to the instruction (Beyotime Biotechnology, Beijing, China). A five-fold dilution of each plasma sample with phosphate buffer saline (PBS, $\mathrm{pH}=7.4$ ) was coated in a microtiter plate (Corning, Corning, NY, USA) at $37^{\circ} \mathrm{C}$ for $2 \mathrm{~h}$. The primary antibody and the secondary antibody were added in turn with incubation at $37^{\circ} \mathrm{C}$ for $1 \mathrm{~h}$. Subsequently, the substrate was added with the absorbance at $450 \mathrm{~nm}$.

Acknowledgments: This study is supported by the Special Fund for Agro-Scientific Research in the Public Interest (201203069-2) and the Fund of Modern Agriculture Industry System Innovation in Beijing City Team (1051-25012090).

Author Contributions: Kui Zhu, Shuangyang Ding and Yifang Cui designed the experiments; Yifang Cui, Yuan Liu and Xiaoye Liu performed the experiments; Kui Zhu and Yifang Cui analyzed the data; Xi Xia contributed reagents/materials/analysis tools; Kui Zhu and Yifang Cui wrote the paper. All authors have read and approved the final manuscript.

Conflicts of Interest: The authors declare no conflict of interest.

\section{References}

1. Bottone, E.J. Bacillus cereus, a volatile human pathogen. Clin. Microbiol. Rev. 2010, 23, 382-398. [CrossRef] [PubMed]

2. Ceuppens, S.; Boon, N.; Uyttendaele, M. Diversity of Bacillus cereus group strains is reflected in their broad range of pathogenicity and diverse ecological lifestyles. FEMS Microbiol. Ecol. 2013, 84, 433-450. [CrossRef] [PubMed]

3. Zhu, K.; Acaroez, U.; Maertlbauer, E. A cellular logic circuit for the detection of bacterial pore-forming toxins. Chem. Commun. 2013, 49, 5198-5200. [CrossRef] [PubMed]

4. Zhu, K.; Didier, A.; Dietrich, R.; Heilkenbrinker, U.; Waltenberger, E.; Jessberger, N.; Martlbauer, E.; Benz, R. Formation of small transmembrane pores: An intermediate stage on the way to Bacillus cereus non-hemolytic enterotoxin (Nhe) full pores in the absence of NheA. Biochem. Biophys. Res. Commun. 2016, 469, 613-618. [CrossRef] [PubMed]

5. Mahler, H.; Pasi, A.; Kramer, J.M.; Schulte, P.; Scoging, A.C.; Bär, W.; Krähenbühl, S. Fulminant liver failure in association with the emetic toxin of Bacillus cereus. N. Engl. J. Med. 1997, 336, 1142-1148. [CrossRef] [PubMed]

6. Naranjo, M.; Denayer, S.; Botteldoorn, N.; Delbrassinne, L.; Veys, J.; Waegenaere, J.; Sirtaine, N.; Driesen, R.B.; Sipido, K.R.; Mahillon, J.; et al. Sudden death of a young adult associated with Bacillus cereus food poisoning. J. Clin. Microbiol. 2011, 49, 4379-4381. [CrossRef] [PubMed]

7. Eurosurveillance Editorial Team. The European Union summary report on trends and sources of zoonoses, zoonotic agents and food-borne outbreaks in 2011. Eurosurveillance 2013, 18. [CrossRef]

8. Wang, S.J.; Duan, H.L.; Zhang, W.; Li, J.W. Analysis of bacterial foodborne disease outbreaks in China between 1994 and 2005. FEMS Immunol. Med. Microbiol. 2007, 51, 8-13. [CrossRef] [PubMed] 
9. Rajkovic, A.; Uyttendaele, M.; Vermeulen, A.; Andjelkovic, M.; Fitz-James, I.; in't Veld, P.; Denon, Q.; Verhe, R.; Debevere, J. Heat resistance of Bacillus cereus emetic toxin, cereulide. Lett. Appl. Microbiol. 2008, 46, 536-541. [CrossRef] [PubMed]

10. Messelhausser, U.; Frenzel, E.; Blochinger, C.; Zucker, R.; Kampf, P.; Ehling-Schulz, A. Emetic Bacillus cereus are more volatile than thought: Recent foodborne outbreaks and prevalence studies in Bavaria (2007-2013). Biomed. Res. Int. 2014, 2014. [CrossRef] [PubMed]

11. Zhou, G.P.; Liu, H.Z.; He, J.; Yuan, Y.M.; Yuan, Z.M. The occurrence of Bacillus cereus, B. thuringiensis and B. mycoides in Chinese pasteurized full fat milk. Int. J. Food Microbiol. 2008, 121, 195-200. [CrossRef] [PubMed]

12. Zhou, G.P.; Zheng, D.S.; Dou, L.; Cai, Q.X.; Yuan, Z.M. Occurrence of psychrotolerant Bacillus cereus group strains in ice creams. Int. J. Food Microbiol. 2010, 137, 143-146. [CrossRef] [PubMed]

13. Marxen, S.; Stark, T.D.; Rutschle, A.; Lucking, G.; Frenzel, E.; Scherer, S.; Ehling-Schulz, M.; Hofmann, T. Depsipeptide intermediates interrogate proposed biosynthesis of cereulide, the emetic toxin of Bacillus cereus. Sci. Rep. 2015, 5. [CrossRef] [PubMed]

14. Makarasen, A.; Yoza, K.; Isobe, M. Higher structure of cereulide, an emetic toxin from Bacillus cereus, and special comparison with valinomycin, an antibiotic from streptomyces fulvissimus. Chem. Asian J. 2009, 4, 688-698. [CrossRef] [PubMed]

15. Shiota, M.; Saitou, K.; Mizumoto, H.; Matsusaka, M.; Agata, N.; Nakayama, M.; Kage, M.; Tatsumi, S.; Okamoto, A.; Yamaguchi, S.; et al. Rapid detoxification of cereulide in Bacillus cereus food poisoning. Pediatrics 2010, 125, E951-E955. [CrossRef] [PubMed]

16. Agata, N.; Ohta, M.; Mori, M.; Isobe, M. A novel dodecadepsipeptide, cereulide, is an emetic toxin of Bacillus cereus. FEMS Microbiol. Lett. 1995, 129, 17-19. [PubMed]

17. Shinagawa, K.; Konuma, H.; Sekita, H.; Sugii, S. Emesis of rhesus monkeys induced by intragastric administration with the HEp-2 vacuolation factor (cereulide) produced by Bacillus cereus. FEMS Microbiol. Lett. 1995, 130, 87-90. [PubMed]

18. Kumari, S.; Sarkar, P.K. Prevalence and characterization of Bacillus cereus group from various marketed dairy products in India. Dairy Sci. Technol. 2014, 94, 483-497. [CrossRef]

19. Zhou, G.P.; Bester, K.; Liao, B.; Yang, Z.S.; Jiang, R.R.; Hendriksen, N.B. Characterization of three Bacillus cereus strains involved in a major outbreak of food poisoning after consumption of fermented black beans (Douchi) in Yunan, China. Foodborne Pathog. Dis. 2014, 11, 769-774. [CrossRef] [PubMed]

20. Karagoz, K.; Adiguzel, G.; Dikbas, N. Emetic and enterotoxin profiles of food-borne Bacillus cereus strains from Turkey. Res. J. Biotechnol. 2015, 10, 44-49.

21. Ronning, H.T.; Asp, T.N.; Granum, P.E. Determination and quantification of the emetic toxin cereulide from Bacillus cereus in pasta, rice and cream with liquid chromatography-tandem mass spectrometry. Food Addit. Contam. Part A Chem. Anal. Control Expo. Risk Assess. 2015, 32, 911-921. [CrossRef] [PubMed]

22. Yim, J.H.; Kim, K.Y.; Chon, J.W.; Kim, D.H.; Kim, H.S.; Choi, D.S.; Choi, I.S.; Seo, K.H. Incidence, antibiotic susceptibility, and toxin profiles of Bacillus cereus sensu lato isolated from Korean fermented soybean products. J. Food Sci. 2015, 80, M1266-M1270. [CrossRef] [PubMed]

23. Ehling-Schulz, M.; Frenzel, E.; Gohar, M. Food-bacteria interplay: Pathometabolism of emetic Bacillus cereus. Front. Microbiol. 2015, 6, 704. [CrossRef] [PubMed]

24. Svensson, B.; Monthan, A.; Shaheen, R.; Andersson, M.A.; Salkinoja-Salonen, M.; Christiansson, A. Occurrence of emetic toxin producing Bacillus cereus in the dairy production chain. Int. Dairy J. 2006, 16, 740-749. [CrossRef]

25. Kim, J.B.; Jeong, H.R.; Park, Y.B.; Kim, J.M.; Oh, D.H. Food poisoning associated with emetic-type of Bacillus cereus in Korea. Foodborne Pathog. Dis. 2010, 7, 555-563. [CrossRef] [PubMed]

26. Ehling-Schulz, M.; Fricker, M.; Grallert, H.; Rieck, P.; Wagner, M.; Scherer, S. Cereulide synthetase gene cluster from emetic Bacillus cereus: Structure and location on a mega virulence plasmid related to Bacillus anthracis toxin plasmid pXOI. BMC Microbiol. 2006, 6. [CrossRef] [PubMed]

27. Zhu, K.; Dietrich, R.; Didier, A.; Acar, G.; Maertlbauer, E. Versatile antibody-sensing Boolean logic for the simultaneous detection of multiple bacterial toxins. Chem. Commun. 2013, 49, 9314-9316. [CrossRef] [PubMed]

28. Ehling-Schulz, M.; Fricker, M.; Scherer, S. Bacillus cereus, the causative agent of an emetic type of food-borne illness. Mol. Nutr. Food Res. 2004, 48, 479-487. [CrossRef] [PubMed] 
29. Yokoyama, K.; Ito, M.; Agata, N.; Isobe, M.; Shibayama, K.; Horii, T.; Ohta, M. Pathological effect of synthetic cereulide, an emetic toxin of Bacillus cereus, is reversible in mice. FEMS Immunol. Med. Microbiol. 1999, 24, 115-120. [CrossRef] [PubMed]

30. Cui, Y.F.; Liu, X.Y.; Dietrich, R.; Martlbauer, E.; Cao, J.; Ding, S.Y.; Zhu, Y. Characterization of Bacillus cereus isolates from local dairy farms in China. FEMS Microbiol. Lett. 2016. [CrossRef]

31. Merzougui, S.; Lkhider, M.; Grosset, N.; Gautier, M.; Cohen, N. Prevalence, PFGE typing, and antibiotic resistance of Bacillus cereus group isolated from food in Morocco. Foodborne Pathog. Dis. 2014, 11, 145-149. [CrossRef] [PubMed]

32. Wehrle, E.; Moravek, M.; Dietrich, R.; Burk, C.; Didier, A.; Martlbauer, E. Comparison of multiplex PCR, enzyme immunoassay and cell culture methods for the detection of enterotoxinogenic Bacillus cereus. J. Microbiol. Methods 2009, 78, 265-270. [CrossRef] [PubMed]

33. Dietrich, R.; Moravek, M.; Burk, C.; Granum, P.E.; Martlbauer, E. Production and characterization of antibodies against each of the three subunits of the Bacillus cereus nonhemolytic enterotoxin complex. Appl. Environ. Microbiol. 2005, 71, 8214-8220. [CrossRef] [PubMed]

34. Ehling-Schulz, M.; Vukov, N.; Schulz, A.; Shaheen, R.; Andersson, M.; Martlbauer, E.; Scherer, S. Identification and partial characterization of the nonribosomal peptide synthetase gene responsible for cereulide production in emetic Bacillus cereus. Appl. Environ. Microbiol. 2005, 71, 105-113. [CrossRef] [PubMed]

35. Bauer, T.; Stark, T.; Hofmann, T.; Ehling-Schulz, M. Development of a stable isotope dilution analysis for the quantification of the Bacillus cereus toxin cereulide in foods. J. Agric. Food Chem. 2010, 58, 1420-1428. [CrossRef] [PubMed]

(C) 2016 by the authors; licensee MDPI, Basel, Switzerland. This article is an open access article distributed under the terms and conditions of the Creative Commons Attribution (CC-BY) license (http://creativecommons.org/licenses/by/4.0/). 